\title{
Genomic inversion drives small colony variant formation and increased virulence in $P$. aeruginosa
}

Sharon Irvine ${ }^{1^{\star}}$, Boyke Bunk ${ }^{2,3^{\star}}$, Hannah K. Bayes ${ }^{1}$, Cathrin Spröer ${ }^{2}$, James P. R. Connolly ${ }^{1}$, Anne Six $^{1}$, Thomas J. Evans ${ }^{1}$, Andrew J. Roe ${ }^{1}$, Jörg Overmann ${ }^{2,3}$ and Daniel Walker ${ }^{1}$

${ }^{1}$ Institute of Infection, Immunity and Inflammation, College of Medical, Veterinary and Life Sciences, University of Glasgow, Glasgow, G12 8QQ, UK.

${ }^{2}$ Leibniz-Institut DSMZ-Deutsche Sammlung von Mikroorganismen und Zellkulturen, Inhiffenstraße 7B, 38124 Braunschweig, Braunschweig, Germany

${ }^{3}$ German Centre of Infection Research (DZIF), Partner site Hannover-Braunschweig, Braunschweig, Germany

*These authors contributed equally.

Corresponding authors: Dr Daniel Walker, E-mail: daniel.walker@glasgow.ac.uk and Professor Jörg Overmann, E-mail: Joerg.Overmann@dsmz.de 


\begin{abstract}
Phenotypic change is a hallmark of bacterial adaptation during chronic infection. In the case of chronic Pseudomonas aeruginosa lung infection in patients with cystic fibrosis, well-characterised phenotypic variants include mucoid and small colony variants (SCVs). It has previously been shown that SCVs can be reproducibly isolated from the murine lung following the establishment of chronic infection with mucoid $P$. aeruginosa strain NH57388A. Here we show, using a combination of singlemolecule real-time (PacBio) and Illumina sequencing that the genetic switch for conversion to the SCV phenotype is a large genomic inversion through recombination between homologous regions of two rRNA operons. This phenotypic conversion is associated with large-scale transcriptional changes distributed throughout the genome. This global rewiring of the cellular transcriptomic output results in changes to normally differentially regulated genes that modulate resistance to oxidative stress, central metabolism and virulence. These changes are of clinical relevance since the appearance of SCVs during chronic infection is associated with declining lung function.
\end{abstract}




\section{Introduction}

Phenotypic variation is a hallmark of adaptation to the host during chronic bacterial infection. There is considerable interest in slow growing subpopulations of bacteria, termed small colony variants (SCVs), due to their association with persistent infections ${ }^{1,2}$. The SCV variant is common to diverse bacteria and is characterised by phenotypes including reduced growth, increased biofilm production $^{3}$, antibiotic resistance and hyperpiliation. SCVs have been described for a wide range of bacterial genera and species including Staphylococcus aureus ${ }^{4,5}$, Staphylococcus epidermidis ${ }^{6}$, Streptococcus $s p^{7,8}$, Enterococcus ${ }^{9}$, Listeria $^{10}$, Burkholderia $^{11}$, Salmonella ${ }^{12}$, Vibrio ${ }^{13}$, Shigella, Brucella $^{14}$, Lactobacillus, Serratia and Neisseria ${ }^{15}$. In the case of Pseudomonas aeruginosa, SCVs are commonly associated with chronic infection of the lung in patients with cystic fibrosis ${ }^{16-21}$.

$P$. aeruginosa is the major proven cause of mortality in patients with CF and chronic infection leads to a progressive decline in pulmonary function and inevitably respiratory failure ${ }^{22}$. Despite intensive anti-pseudomonal chemotherapy greatly improving the prognosis for CF patients ${ }^{23}$, the current median age at death for CF patients is around thirty years in developed countries ${ }^{24}$. The frequent failure of antibiotic therapy and host defences to eradicate $P$. aeruginosa from the CF lung is thought to be largely due to the increased antibiotic tolerance when growing in the biofilm state and the appearance of mucoid phenotyic variants that are a hallmark of adaptation in the chronically infected lung. A further complicating factor is the appearance of highly adherent SCVs that are adept at biofilm formation ${ }^{18,19,25}$. P. aeruginosa SCVs may display high intracellular C-di-GMP levels ${ }^{19,20,26-}$ ${ }^{28}$, enhanced biofilm formation, high fimbrial expression, repression of flagellar genes, resistance to phagocytosis and enhanced antibiotic resistance. Most importantly, the appearance of SCVs in the CF lung correlates with poor patient clinical outcome ${ }^{11,17,29-31}$.

There are a range of genetic changes that have been shown to be responsible for the phenotypic switch to the SCV phenotype in P. aeruginosa, including mutations in the Wsp system and $y f i B N R$ operon that form part of the c-di-GMP regulatory system in $P$. aeruginosa ${ }^{32-36}$. However, identification of the major clinically relevant pathways of conversion to the SCV phenotype are complicated by the unstable phenotype displayed by many SCVs with reversion to a normal colony phenotype frequently observed preventing successful comparative genetic studies on clinical SCVs and their closely related parent strains. In the case of $S$. aureus, which also forms clinically relevant SCVs, recent work has shown that a reversible large scale chromosomal inversion is the genetic basis of the switch between a normal colony and SCV isolated from the same patient ${ }^{37}$. In addition, S. aureus SCVs, which are commonly isolated from the CF lung, are highly resistant to oxidative stress, suggesting that conversion to the SCV phenotype may be an adaptation to environment present in chronically inflamed host tissue ${ }^{38}$.

In this work, we have determined the genetic basis of phenotypic conversion from the mucoid to the SCV phenotype for SCVs isolated from the chronic lung infection model described by Bayes et al $2016^{39}$. For two SCVs isolated from this work, we have shown through a combination of singlemolecule real-time (SMRT, Pacific Biosciences) and Illumina sequencing that a large and stable 
chromosomal inversion accompanies conversion to the SCV phenotype. Genome inversion is accompanied by transcriptional changes to a large number of genes that most notably includes downregulation of a number of genes encoding metabolic enzymes, DNA repair proteins and heat shock proteins and the upregulation of genes encoding proteins involved in the response to oxidative stress. The absence of other obvious genetic change suggests that this chromosomal inversion is the genetic basis of conversion to the SCV phenotype.

\section{Results}

$P$. aeruginosa SCVs are commonly isolated from patients with cystic fibrosis and have been isolated in vitro as well as from experimental infection models following aminoglycoside treatment ${ }^{18,31,40}$. The work of Bayes et al describes the isolation and partial characterisation of SCVs isolated from a chronic murine $P$. aeruginosa lung infection model ${ }^{39}$. In this model, animals were inoculated with $P$. aeruginosa strain NH57388A (NHMuc) a mucoid clinical isolate, embedded in agar beads. NHMuc has a known mutation in the gene encoding the anti-sigma factor MucA, that results in alginate overproduction ${ }^{41,42}$. Recovered bacteria from lung homogenate samples display two distinct colony morphologies: typical large mucoid colonies identical in morphology to the inoculating strain and SCVs. Mucoid colonies were evident after 24 hours of growth on agar plates at $37^{\circ} \mathrm{C}$ with SCVs visible only after 48 hours of growth on agar plates ${ }^{39}$.

To understand the genetic basis of this phenotypic change, we initially performed Illumina HiSeq whole-genome sequencing and genomic comparison between $\mathrm{NH}$ and two separate SCVs (SCVJan and SCVFeb) isolated from independent in vivo experiments. However, despite their gross phenotypic differences, this analysis failed to identify any genetic differences between the SCVs and the parent strain.

Next, we utilised the ultra-long reads produced by single-molecule real-time PacBio sequencing to attempt to identify any large scale genome rearrangements that could drive conversion to the SCV phenotype. Using this technique we identified a large scale genomic inversion accompanying conversion from the parent mucoid to SCV phenotype in both SCVJan and SCVFeb. Closer inspection of the genome sequence identified the start and end points of the inversion which for both SCVJan and SCVFeb begins at the first rRNA operon (0.72 Mbp) and ends at the third rRNA operon (5.21 Mbp). Exact chromosomal breakpoints were identified in the corresponding 16S rRNA genes by performing a MAUVE breakpoint analysis (Figure 1). Furthermore, genome analysis revealed a 250 bp shortened 16S rRNA gene $\left(16 S_{t}\right)$ in both SCV strains, which is reflected in the reduced genome sizes of the SCVs (SCVJan 6,213,026, SCVFeb 6,213,029; Figure 2a) compared to the parent strain, NHmuc $(6,213,276$ bp; Figure $2 b)$. There were no further differences in the number of protein coding genes (5619), rRNAs (12) or tRNAs (57) between SCVs and the parent strain. No SNPs could be identified in protein coding genes. A similar genome inversion was not identified (using a PCR based strategy) in a mucoid strain (NHMucJan) that was phenotypically identical to the parent strain (NHMuc) and isolated from the same chronic infection model as SCVJan 
(Figure S1). Interestingly, comparison of the SCVJan and SCVFeb genomes with that of an SCV (SCV20265) isolated from a CF patient ${ }^{43}$, which has recently been sequenced by PacBio sequencing, revealed an almost identical chromosomal inversion. However, in the case of SCV20265 the inversion was not accompanied by truncation of the 16S rRNA gene in the third rRNA operon (Figure 1).

\section{Transcriptional and phenotypic changes on conversion to the SCV phenotype}

To determine the transcriptional changes associated with conversion to the SCV phenotype, we performed RNA sequencing (RNA-Seq) analysis of the parent strain, NHMuc, and two SCV strains grown in LB broth. Initial analysis showed that SCVJan and SCVFeb have highly similar gene expression profiles that are distinct from that of NHMuc. RNA-Seq data for all strains was collected in triplicate and data for SCVJan and SCVFeb were combined to compare with NHMuc. Relative to NHMuc, 190 genes showed >2-fold upregulation and 364 genes showed >2-fold downregulation in SCVJan/SCVFeb (Table S1). Interestingly, the transcriptional changes associated with genomic inversion and that drive conversion to the SCV phenotype are not restricted to genes close to or within the inversion breakpoints, with major upregulated and downregulated genes distributed relatively evenly throughout the genome (Figure 3).

Major functional classes of genes downregulated in SCVJan/SCVFeb include those involved in energy metabolism, amino acid and protein biosynthesis, DNA replication and recombination and cell wall/LPS/capsule biosynthesis, which together are consistent with the slow growth rate observed for SCVs. Notably, genes encoding heat shock proteins and other molecular chaperones (IbpA, GrpE, HtpG, ClpB, DnaK, GroES, DnaJ and ClpX) are highly represented among the most strongly downregulated genes in the SCVs (Table 1). Conversely, genes that function in the response to oxidative stress and those that encode secreted virulence factors are largely upregulated in SCVJan/SCVFeb. Indeed, five of the ten most highly upregulated genes in SCVJan/SCVFeb are those associated with the response to oxidative stress (Table 2). Highly upregulated oxidative stress genes include, kat $A^{44}$ which encodes the major catalase of $P$. aeruginosa, ahpB, ahpC and ahpF ${ }^{45-}$ 47 that encode subunits of alkyl hydroperoxide reductase and trxB2 that encodes thioredoxin reductase $2^{48-50}$. Consistent with the observed transcriptional changes, catalase activity was strongly increased in SCVJan relative to NHMuc (Figure 4A).

Genes encoding a number of secreted virulence factors such as the proteases LasA, LasB and $A p r A$, the frucose-binding lectin $\operatorname{LecB}^{51}$ and the chitin binding protein $C b p D$ and chitinase $C h i C^{52}$ were also highly upregulated. Similarly genes encoding hydrogen cyanide synthase and a number of enzymes that function in phenazine biosynthesis are also upregulated (Table S1) ${ }^{28,53-57}$. Phenazines have previously been shown to enhance killing of Caenorhabditis elegans by $P$. aeruginosa ${ }^{58}$. The apparent increase in the production of virulence factors by SCVJan/SCVFeb relative to NHMuc suggests increased virulence of the SCV. To directly test this we used an infection model based on infection of the murine macrophage cell line J774A.1. Cell death of J774A.1 through 
LDH release was measured at 4 and 10 hours post infection with NHMuc and SCVJan. At 4 hours, levels of LDH release were similar for NHMuc and SCVJan, whereas at 10 hours LDH release was significantly increased for SCVJan; 96\% vs 38\%, p < 0.0001 (Figure 4B).

To determine if the increased virulence of the SCV observed against a murine cell line translated to increased virulence in an animal model of infection, we utilised an invertebrate model of infection utilising the larva of the wax moth Galleria mellonella. Similar to the macrophage infection assay, SCVJan displayed increased virulence in the G. mellonella infection model. Mortality of larvae was measured at 24, 48 and 72 hours post infection. No significant differences in mortality were detected at 24 or 48 hours, whereas at 72 hours \% mortality was $86 \%$ and 63\% $(p<0.04)$ for SCVJan and NHMuc infected larvae, respectively (Figure 4C). Data from both infection models shows that SCVJan shows increased virulence, relative to NHMuc, which is consistent with the phenotype of SCVs obtained from the human host. ${ }^{31,59}$

\section{Discussion}

In this work we show that $P$. aeruginosa SCVs isolated from a chronic murine lung infection model show a general upregulation of virulence associated genes, relative to the mucoid parent strain, and increased virulence which may begin to explain the link between the appearance of SCVs in chronic lung infection and the associated decline in lung function ${ }^{60}$. In addition, the upregulation of genes that mediate the response to oxidative stress immediately suggests why the isolated SCVs are rapidly selected for in a chronic infection model in which the host immune system is strongly activated.

A key strength of our study was the availability of the parent strain used to establish infection for sequencing. This allowed a meaningful comparative genetic analysis to be performed enabling the determination for the genetic basis of conversion to the SCV phenotype. Surprisingly, SNPs and INDELS were not identified in the SCV genome by Illumina sequencing and single-molecule realtime sequencing was subsequently used to show that the two sequenced SCVs carried a large genomic inversion within 16S rRNA genes. Interestingly, the transcriptional changes associated with genomic inversion are not restricted to genes close to or within the inversion breakpoints, but distributed relatively evenly throughout the genome. Instead the major changes in gene expression are largely restricted to specific functional classes of genes including those that mediate the response to oxidative stress, virulence, DNA repair and recombination, the chaperone network and metabolism. This global rewiring of the cellular transcriptomic output results in concerted transcriptional changes to these normally differentially regulated genes. However, the mechanisms that underlie these transcriptional changes are not clear. A recent study in $E$. coli suggests that position effects on gene expression may be due to local differences in chromosomal structuring and organization, with DNA gyrase playing an important role at certain high activity locations. Further studies will be needed to clarify such positional effects on gene expression in the SCV studied here ${ }^{61}$.

The mechanistic details of how the observed genomic inversion lead to the coordinated expression changes observed here is currently not known, but the observation that a clinical SCV 
strain (SCV20265) obtained from the lung of a CF patient strain recently sequenced using the same strategy of combining SMRT and Illumina sequencing possesses a similar 16S rRNA based inversion, indicates that this inversion may be a highly clinically relevant route to the SCV phenotype (Figure 1$)^{43}$. Other large scale genome rearrangements including large chromosomal inversions have previously been described in $P$. aeruginosa but these were not associated with conversion to the SCV phenotype ${ }^{62,63}$. A reversible genomic inversion has also recently been shown to mediate the reversible conversion between normal colony and SCV phenotypes in S. aureus ${ }^{37}$. However, in the case of the SCVs isolated in our work the SCV phenotype is stable and revertants to the parent phenotype were not observed. A possible explanation for this observation is that a number of genes encoding proteins involved in DNA repair and recombination, including RecA, are downregulated in the SCV relative to the parent strain (Table 1).

In conclusion, we have shown that a $P$. aeruginosa SCV originated in the lungs of an animal with chronic colonization appears to result from a large chromosomal inversion and associated largescale transcriptional changes. SCVs have a clear selective advantage in the context of the CF lung, and a better understanding of the drivers that produce the genomic rearrangement observed in this study may provide alternative therapeutic approaches to prevent the appearance of such damaging phenotypic variants.

\section{Materials and Methods}

Genome assembly and annotation. Purified bacterial genomic DNA was prepared for sequencing on Illumina HiSeq using QIAGEN DNeasy Blood and Tissue Kit as per manufacturers protocol. Sequencing and initial bioinformatics were performed in the Centre for Genomic Research, University of Liverpool. Sequencing reads were mapped to the corresponding reference genome (annotated NH strain). SMRTbell ${ }^{\mathrm{TM}}$ template libraries were prepared according to the instructions from Pacific Biosciences, Menlo Park, CA, USA, following the Procedure \& Checklist Greater than $10 \mathrm{~kb}$ Template Preparation and Sequencing. Briefly, for preparation of $10 \mathrm{~kb}$ libraries $\sim 10 \mu \mathrm{g}$ genomic DNA isolated from SCVJan, SCVFeb and NHmuc was sheared using g-tubes ${ }^{\mathrm{TM}}$ from Covaris, Woburn, MA, USA according to the manufacturer's instructions. 5-10 $\mu$ g sheared genomic DNA was end-repaired and ligated overnight to hairpin adapters applying components from the DNA/Polymerase Binding Kit P4 from Pacific Biosciences, Menlo Park, CA, USA. Reactions were carried out according to the manufacturer's instructions. SMRTbell ${ }^{\mathrm{TM}}$ template was Exonuclease treated for removal of incompletely formed reaction products. Conditions for annealing of sequencing primers and binding of polymerase to purified SMRTbell ${ }^{\mathrm{TM}}$ template were assessed with the Calculator in RS Remote, PacificBiosciences, Menlo Park, CA, USA. SMRT sequencing was carried out on the PacBio RSII (PacificBiosciences, Menlo Park, CA, USA) taking one 180-minutes movie for each SMRT cell. In total 6, 6 and 5 SMRT cells were run respectively. Data from each SMRT Cell was assembled independently using the "RS_HGAP_Assembly.3" protocol included in SMRTPortal version 2.3.0 using default parameters. Each assembly revealed the fully resolved 
chromosome in one single contig. Each chromosome was circularized independently, particularly artificial redundancies at the ends of the contigs were removed and all chromosomes were additionally adjusted to $d n a A$ as the first gene. Validity of each assembly was checked using the "RS_Bridgemapper.1" protocol. For the purpose of this study it has been confirmed for each of the (repetitive) rRNA operons that enough uniquely mapping long read exist spanning the whole repeat structure. Finally, each genome was error-corrected by a mapping of Illumina reads (paired end reads, $100 \mathrm{bp}$ ) onto finished genomes using $B W A^{64}$ with subsequent variant calling using VarScan ${ }^{65}$. A consensus concordance of QV60 could be confirmed for all of the three genomes. Finally, all genomes were annotated using Prokka 1.866. All genome sequences were deposited in NCBI GenBank under Accession Numbers CP013477, CP013478 and CP013479. Illumina short read data has been deposited at EMBL-EBI ENA database under study number PRJEB12456. The shortened 16S rRNA gene for strains SCVJan and SCVFeb was confirmed by PacBio assembly as well as BWA mapping of Illumina reads against the final chromosome showing uniquely mapped reads only at that genome position (data not shown).

Transcriptome analysis. Ribonucleic acid isolation of the samples was performed in triplicate. Bacterial suspensions were grown to early stationary phase to an $\mathrm{OD}_{600}$ of 1.8 in LB broth at 37 in a shaking incubator. $2 \mathrm{ml}$ of each suspension was pelleted at $12000 \mathrm{~g}$ for 10 minutes. RNA was extracted from samples using a bead beating/chloroform extraction method as previously described $^{67}$. The samples were digested with DNAse I for 1 hour. Bacterial RNA was enriched using MICROBEnrich (Life Technologies) as per protocol. Ribosomal RNA was depleted using Ribo-Zero Magnetic Gold Kit (Epidemiology) (Epicentre) as per manufacturers protocol. The precipitated sample was resuspended in $20 \mu \mathrm{l}$ of RNAse free water. The concentration of RNA was initially determined using Nanodrop followed by an Agilent Bioanalyser. cDNA was generated by using the methods from the Superscript Double-Stranded cDNA Synthesis Kit (Invitrogen) per manufacturer's instructions.

Transcriptome analysis was performed using CLC workbench version 7.0 and significantly upregulated and downregulated genes in SCVJan/SCVFeb vs NHMuc were identified using the CLC software package. Transcriptome data was deposited at EMBL-EBI ENA database under study number PRJEB12456.

PCR Genomic DNA was extracted from $1.5 \mathrm{ml}$ of bacterial culture using the GenElute Bacterial Genomic DNA Kit (Sigma). Extraction was performed following manufacturer recommendations and DNA was eluted into $100 \mu \mathrm{L}$ of Elute Solution. PCR detection of the inversion was performed with the KAPA polymerase (KAPA Biosystems, KAPA Long-range HotStart PCR Kit) following the manufacturer's protocol. Two sets of primers were used: birA-F/yedZ-R and birA-F/tyrZ-R (birA-F: CTCACCGGAGTGGAATC, yedZ-R: TGAGCGCTTACTGCGTGTTCATCCTGG and tyrZ-R: CCATACCGTGCTTATTAATAAGC) with the genomic DNAs of the mucoid and SCV strains recovered from animals amplifying a fragment of 6262 bp or $6960 \mathrm{bp}$, respectively. 
Galleria mellonella infection model. Larvae were stored on wood chips at $4{ }^{\circ} \mathrm{C}$. Overnight cultures of bacterial strains were grown in LB broth, diluted 1:100 in the same medium and grown to an $\mathrm{OD}_{600}$ of 0.3 to 0.4 as previously described ${ }^{68,69}$. Cultures were centrifuged and pellets were washed twice and resuspended in $10 \mathrm{mM}$ PBS to an OD600 of 0.1. Serial 10-fold dilutions were made in PBS. Five-microliter aliquots of the serial dilutions were injected using a Hamilton syringe into $G$. mellonella larvae, via the hindmost left proleg as previously described ${ }^{70}$. Ten larvae were injected per dilution for each Pseudomonas strain tested. Larvae were incubated in $10-\mathrm{cm}$ plates at $37^{\circ} \mathrm{C}$ and the number of dead larvae scored 1 to 4 days after infection. For each strain, data from 3 independent experiments were combined. Larvae were considered dead when they displayed no movement in response to touch. A negative control was used in each experiment to monitor the killing due to physical injury or infection by pathogenic contaminants. Time to death was monitored every $24 \mathrm{~h}$ post infection. In any instance where more than one control larvae died in any given experiment, the data from infected larvae were not used.

LDH Release/Cytotoxicity Assay. To investigate the effect of the $P$. aeruginosa strains on macrophages, we infected the J774A.1 cells with NH and SCV. Bacteria were grown for 17 hours to stationary phase in LB broth at $37^{\circ} \mathrm{C}$. Immediately prior to infection, the bacteria were diluted to exponential growth phase with culture medium lacking phenol red and the concentration determined by measuring the optical density at $600 \mathrm{~nm}$. Cells were grown, washed and infected as previously documented ${ }^{71}$. Cells were infected with test organisms and incubated for $4 \mathrm{hr}$ and $10 \mathrm{hr}$. Lactate dehydrogenase release was determined using the Cytotox 96 cytotoxicity assay kit (Promega USA) as per manufacturers protocol.

Catalase Activity Assay. Overnight cultures of bacterial strains were grown in LB broth, diluted $1: 100$ in the same medium and grown to an $\mathrm{OD}_{600}$ of 0.4 . Catalase standards were prepared as per the manufacturers protocol using the OxiSelect ${ }^{\mathrm{TM}}$ Catalase Activity Assay Kit, Colorimetric (Cell Biolabs, inc). $20 \mu$ of each serial dilution of overnight culture were added to 3 wells in a 96 well plate to allow for average readings for each sample. Plate absorbance was read at 520nm using FLUOstar Optima plate reader (BMG UK).

Accessibility of biological resources. SCVs used in this study have been deposited at DSMZ under DSM 100776 - 100778.

\section{Acknowledgements}

This work was funded by an MRC SCP3 fellowship grant G1000419 and by grant 8000-105-3 of the German Federal Ministry of Science and Education through the German Centre of Infection Research (DZIF) to J.O. We thank Simone Severitt and Nicole Mrotzek at the Leibniz Institute DSMZ, Germany, for excellent technical assistance. 


\section{Author Contributions Statement}

S.I, D.W, B.B and J.O conceived and designed the experiments and analysis. S.I, B.B, C.S performed the experiments. S.I, B.B, J.P.R.C and A.J.R analysed the data. T.J.E and H.K.B supplied novel reagents. D.W and S.I wrote the manuscript.

\section{Competing financial interests}

The authors declare no competing financial interests. 


\section{References}

1. Proctor, R. A. et al. Small colony variants: a pathogenic form of bacteria that facilitates persistent and recurrent infections. Nat. Rev. Microbiol. 4, 295-305 (2006).

2. Grant, S. S. \& Hung, D. T. Persistent bacterial infections, antibiotic tolerance, and the oxidative stress response. Virulence 4, 273-83 (2013).

3. Déziel, E., Comeau, Y. \& Villemur, R. Initiation of biofilm formation by Pseudomonas aeruginosa 57RP correlates with emergence of hyperpiliated and highly adherent phenotypic variants deficient in swimming, swarming, and twitching motilities. J. Bacteriol. 183, 1195-204 (2001).

4. Mirani, Z. A., Aziz, M. \& Khan, S. I. Small colony variants have a major role in stability and persistence of Staphylococcus aureus biofilms. J. Antibiot. (Tokyo). 68, 98-105 (2014).

5. Brouillette, E., Martinez, A., Boyll, B. J., Allen, N. E. \& Malouin, F. Persistence of a Staphylococcus aureus small-colony variant under antibiotic pressure in vivo. FEMS Immunol. Med. Microbiol. 41, 35-41 (2004).

6. Maduka-Ezeh, A. N. et al. Antimicrobial susceptibility and biofilm formation of Staphylococcus epidermidis small colony variants associated with prosthetic joint infection. Diagn. Microbiol. Infect. Dis. 74, 224-9 (2012).

7. Allegrucci, M. \& Sauer, K. Formation of Streptococcus pneumoniae non-phase-variable colony variants is due to increased mutation frequency present under biofilm growth conditions. J. Bacteriol. 190, 6330-9 (2008).

8. Zbinden, A. et al. Characterization of Streptococcus tigurinus small-colony variants causing prosthetic joint infection by comparative whole-genome analyses. J. Clin. Microbiol. 52, 467-74 (2014).

9. Wellinghausen, N. et al. Characterization of clinical Enterococcus faecalis small-colony variants. J. Clin. Microbiol. 47, 2802-11 (2009).

10. Rea, R., Hill, C. \& Gahan, C. G. M. Listeria monocytogenes PerR mutants display a smallcolony phenotype, increased sensitivity to hydrogen peroxide, and significantly reduced murine virulence. Appl. Environ. Microbiol. 71, 8314-22 (2005).

11. Pinto-de-Oliveira, A. et al. 109 The Burkholderia cepacia small colony variants (SCV) are a more pathogenic bacterial form that may facilitate persistent respiratory infections in CF patients. J. Cyst. Fibros. 12, S76 (2013).

12. Cano, D. A., Pucciarelli, M. G., Martínez-Moya, M., Casadesús, J. \& García-del Portillo, F. Selection of small-colony variants of Salmonella enterica serovar typhimurium in nonphagocytic eucaryotic cells. Infect. Immun. 71, 3690-8 (2003).

13. Roggenkamp, A., Hoffmann, H. \& Hornef, M. W. Growth control of small-colony variants by genetic regulation of the hemin uptake system. Infect. Immun. 72, 2254-62 (2004).

14. Jacob, J., Hort, G. M., Overhoff, P. \& Mielke, M. E. A. In vitro and in vivo characterization of smooth small colony variants of Brucella abortus S19. Microbes Infect. 8, 363-71 (2006).

15. Morton, H. E. \& Shoemaker, J. The Identification of Neisseria gonorrhoeae by Means of Bacterial Variation and the Detection of Small Colony Forms in Clinical Material. J. Bacteriol. 50, 585-7 (1945).

16. Haussler, S. Highly adherent small-colony variants of Pseudomonas aeruginosa in cystic fibrosis lung infection. J. Med. Microbiol. 52, 295-301 (2003).

17. Kahl, B. et al. Persistent infection with small colony variant strains of Staphylococcus aureus in patients with cystic fibrosis. J. Infect. Dis. 177, 1023-9 (1998). 
18. Haussler, S. Highly adherent small-colony variants of Pseudomonas aeruginosa in cystic fibrosis lung infection. J. Med. Microbiol. 52, 295-301 (2003).

19. Häussler, S. Biofilm formation by the small colony variant phenotype of Pseudomonas aeruginosa. Environ. Microbiol. 6, 546-51 (2004).

20. Malone, J. G. et al. YfiBNR mediates cyclic di-GMP dependent small colony variant formation and persistence in Pseudomonas aeruginosa. PLoS Pathog. 6, e1000804 (2010).

21. Besier, S. et al. Prevalence and clinical significance of Staphylococcus aureus small-colony variants in cystic fibrosis lung disease. J. Clin. Microbiol. 45, 168-72 (2007).

22. O'Sullivan, B. P. \& Freedman, S. D. Cystic fibrosis. Lancet 373, 1891-904 (2009).

23. Folkesson, A. et al. Adaptation of Pseudomonas aeruginosa to the cystic fibrosis airway: an evolutionary perspective. Nat. Rev. Microbiol. 10, 841-51 (2012).

24. CF Registry - Annual Data Reports - Cystic Fibrosis Trust. at <http://www.cysticfibrosis.org.uk/research-care/uk-cf-registry/cf-registry-reports>

25. Webb, J. S., Lau, M. \& Kjelleberg, S. Bacteriophage and phenotypic variation in Pseudomonas aeruginosa biofilm development. J. Bacteriol. 186, 8066-73 (2004).

26. Malone, J. G. et al. YfiBNR mediates cyclic di-GMP dependent small colony variant formation and persistence in Pseudomonas aeruginosa. PLoS Pathog. 6, e1000804 (2010).

27. Starkey, M. et al. Pseudomonas aeruginosa rugose small-colony variants have adaptations that likely promote persistence in the cystic fibrosis lung. J. Bacteriol. 191, 3492-503 (2009).

28. Wang, D. et al. Adaptation genomics of a small-colony variant in a Pseudomonas chlororaphis 30-84 biofilm. Appl. Environ. Microbiol. 81, 890-9 (2015).

29. Besier, S. et al. Prevalence and clinical significance of Staphylococcus aureus small-colony variants in cystic fibrosis lung disease. J. Clin. Microbiol. 45, 168-72 (2007).

30. Wolter, D. J. et al. Staphylococcus aureus small-colony variants are independently associated with worse lung disease in children with cystic fibrosis. Clin. Infect. Dis. 57, 38491 (2013).

31. von Götz, F. et al. Expression analysis of a highly adherent and cytotoxic small colony variant of Pseudomonas aeruginosa isolated from a lung of a patient with cystic fibrosis. $J$. Bacteriol. 186, 3837-47 (2004).

32. Deretic, V., Govan, J. R., Konyecsni, W. M. \& Martin, D. W. Mucoid Pseudomonas aeruginosa in cystic fibrosis: mutations in the muc loci affect transcription of the algR and algD genes in response to environmental stimuli. Mol. Microbiol. 4, 189-96 (1990).

33. Cooper, V. S., Staples, R. K., Traverse, C. C. \& Ellis, C. N. Parallel evolution of small colony variants in Burkholderia cenocepacia biofilms. Genomics 104, 447-452 (2014).

34. Malone, J. G. et al. The YfiBNR signal transduction mechanism reveals novel targets for the evolution of persistent Pseudomonas aeruginosa in cystic fibrosis airways. PLoS Pathog. 8, e1002760 (2012).

35. Hickman, J. W., Tifrea, D. F. \& Harwood, C. S. A chemosensory system that regulates biofilm formation through modulation of cyclic diguanylate levels. Proc. Natl. Acad. Sci. U. S. A. 102, 14422-7 (2005).

36. D'Argenio, D. A., Calfee, M. W., Rainey, P. B. \& Pesci, E. C. Autolysis and autoaggregation in Pseudomonas aeruginosa colony morphology mutants. J. Bacteriol. 184, 6481-9 (2002).

37. Cui, L., Neoh, H., Iwamoto, A. \& Hiramatsu, K. Coordinated phenotype switching with largescale chromosome flip-flop inversion observed in bacteria. Proc. Natl. Acad. Sci. U. S. A. 109, E1647-56 (2012).

38. Painter, K. L. et al. Staphylococcus aureus adapts to oxidative stress by producing $\mathrm{H} 2 \mathrm{O} 2-$ 
resistant small colony variants via the SOS response. Infect. Immun. 83, 1830-1844 (2015).

39. Bayes, H. K., Ritchie, N., Irvine, S. \& Evans, T. J. A murine model of early Pseudomonas aeruginosa lung disease with transition to chronic infection. Sci. Rep. 6, 35838 (2016).

40. Schaaff, F., Bierbaum, G., Baumert, N., Bartmann, P. \& Sahl, H.-G. Mutations are involved in emergence of aminoglycoside-induced small colony variants of Staphylococcus aureus. Int. J. Med. Microbiol. 293, 427-35 (2003).

41. Hoffmann, N. et al. Novel mouse model of chronic Pseudomonas aeruginosa lung infection mimicking cystic fibrosis. Infect. Immun. 73, 2504-14 (2005).

42. Boucher, J. C., Yu, H., Mudd, M. H. \& Deretic, V. Mucoid Pseudomonas aeruginosa in cystic fibrosis: characterization of muc mutations in clinical isolates and analysis of clearance in a mouse model of respiratory infection. Infect. Immun. 65, 3838-46 (1997).

43. Eckweiler, D., Bunk, B., Spröer, C., Overmann, J. \& Häussler, S. Complete Genome Sequence of Highly Adherent Pseudomonas aeruginosa Small-Colony Variant SCV20265. Genome Announc. 2, e01232-13 (2014).

44. Lee, J.-S., Heo, Y.-J., Lee, J. K. \& Cho, Y.-H. KatA, the major catalase, is critical for osmoprotection and virulence in Pseudomonas aeruginosa PA14. Infect. Immun. 73, 4399403 (2005).

45. Ochsner, U. A., Vasil, M. L., Alsabbagh, E., Parvatiyar, K. \& Hassett, D. J. Role of the Pseudomonas aeruginosa oxyR-recG Operon in Oxidative Stress Defense and DNA Repair: OxyR-Dependent Regulation of katB-ankB, ahpB, and ahpC-ahpF. J. Bacteriol. 182, 45334544 (2000).

46. Ochsner, U. A., Vasil, M. L., Alsabbagh, E., Parvatiyar, K. \& Hassett, D. J. Role of the Pseudomonas aeruginosa oxyR-recG operon in oxidative stress defense and DNA repair: OxyR-dependent regulation of katB-ankB, ahpB, and ahpC-ahpF. J. Bacteriol. 182, 4533-44 (2000).

47. Vinckx, T., Matthijs, S. \& Cornelis, P. Loss of the oxidative stress regulator OxyR in Pseudomonas aeruginosa PAO1 impairs growth under iron-limited conditions. FEMS Microbiol. Lett. 288, 258-65 (2008).

48. Chung, J.-S. et al. Interaction domain on thioredoxin for Pseudomonas aeruginosa 5'adenylylsulfate reductase. J. Biol. Chem. 284, 31181-9 (2009).

49. Hishinuma, S., Ohtsu, I., Fujimura, M. \& Fukumori, F. OxyR is involved in the expression of thioredoxin reductase TrxB in Pseudomonas putida. FEMS Microbiol. Lett. 289, 138-45 (2008).

50. Zhou, A. et al. Hydrogen peroxide-induced oxidative stress responses in Desulfovibrio vulgaris Hildenborough. Environ. Microbiol. 12, 2645-57 (2010).

51. Tielker, D. et al. Pseudomonas aeruginosa lectin LecB is located in the outer membrane and is involved in biofilm formation. Microbiology 151, 1313-23 (2005).

52. Frederiksen, R. F. et al. Bacterial chitinases and chitin-binding proteins as virulence factors. Microbiology 159, 833-47 (2013).

53. Mavrodi, D. V et al. Functional analysis of genes for biosynthesis of pyocyanin and phenazine-1-carboxamide from Pseudomonas aeruginosa PAO1. J. Bacteriol. 183, 6454-65 (2001).

54. Lau, G. W., Ran, H., Kong, F., Hassett, D. J. \& Mavrodi, D. Pseudomonas aeruginosa pyocyanin is critical for lung infection in mice. Infect. Immun. 72, 4275-8 (2004).

55. Wang, D. et al. Adaptation genomics of a small-colony variant in a Pseudomonas chlororaphis 30-84 biofilm. Appl. Environ. Microbiol. 81, 890-9 (2015).

56. Pessi, G. et al. The global posttranscriptional regulator RsmA modulates production of 
virulence determinants and $\mathrm{N}$-acylhomoserine lactones in Pseudomonas aeruginosa. $\mathrm{J}$. Bacteriol. 183, 6676-83 (2001).

57. Sanchez, P. Fitness of in vitro selected Pseudomonas aeruginosanalB and nfxB multidrug resistant mutants. J. Antimicrob. Chemother. 50, 657-664 (2002).

58. Cezairliyan, B. et al. Identification of Pseudomonas aeruginosa phenazines that kill Caenorhabditis elegans. PLoS Pathog. 9, e1003101 (2013).

59. Sabra, W., Haddad, A. M. \& Zeng, A.-P. Comparative physiological study of the wild type and the small colony variant of Pseudomonas aeruginosa 20265 under controlled growth conditions. World J. Microbiol. Biotechnol. 30, 1027-36 (2014).

60. Small Colony Variants of Pseudomonas aeruginosa in Cystic Fibrosis. at <http://cid.oxfordjournals.org/content/29/3/621.full.pdf>

61. Bryant, J. A., Sellars, L. E., Busby, S. J. W. \& Lee, D. J. Chromosome position effects on gene expression in Escherichia coli K-12. Nucleic Acids Res. 42, 11383-92 (2014).

62. Stover, C. K. et al. Complete genome sequence of Pseudomonas aeruginosa PAO1, an opportunistic pathogen. Nature 406, 959-64 (2000).

63. Römling, U., Schmidt, K. D. \& Tümmler, B. Large genome rearrangements discovered by the detailed analysis of 21 Pseudomonas aeruginosa clone $\mathrm{C}$ isolates found in environment and disease habitats. J. Mol. Biol. 271, 386-404 (1997).

64. Li, H. \& Durbin, R. Fast and accurate short read alignment with Burrows-Wheeler transform. Bioinformatics 25, 1754-60 (2009).

65. Koboldt, D. C. et al. VarScan 2: somatic mutation and copy number alteration discovery in cancer by exome sequencing. Genome Res. 22, 568-76 (2012).

66. Seemann, T. Prokka: rapid prokaryotic genome annotation. Bioinformatics 30, 2068-9 (2014).

67. Lim, Y. W. et al. Metagenomics and metatranscriptomics: Windows on CF-associated viral and microbial communities. J. Cyst. Fibros. 12, 154-164 (2013).

68. Ramarao, N., Nielsen-Leroux, C. \& Lereclus, D. The insect Galleria mellonella as a powerful infection model to investigate bacterial pathogenesis. J. Vis. Exp. e4392 (2012). doi:10.3791/4392

69. Miyata, S., Casey, M., Frank, D. W., Ausubel, F. M. \& Drenkard, E. Use of the Galleria mellonella Caterpillar as a Model Host To Study the Role of the Type III Secretion System in Pseudomonas aeruginosa Pathogenesis. Infect. Immun. 71, 2404-2413 (2003).

70. Jander, G., Rahme, L. G. \& Ausubel, F. M. Positive correlation between virulence of Pseudomonas aeruginosa mutants in mice and insects. J. Bacteriol. 182, 3843-5 (2000).

71. Hauser, A. R. \& Engel, J. N. Pseudomonas aeruginosa induces type-III-secretion-mediated apoptosis of macrophages and epithelial cells. Infect. Immun. 67, 5530-7 (1999).

72. Carver, T., Thomson, N., Bleasby, A., Berriman, M. \& Parkhill, J. DNAPlotter: circular and linear interactive genome visualization. Bioinformatics 25, 119-20 (2009). 
bioRxiv preprint doi: https://doi.org/10.1101/356386; this version posted July 9,2018 . The copyright holder for this preprint (which was not certified by peer review) is the author/funder, who has granted bioRxiv a license to display the preprint in perpetuity. It is made available under aCC-BY 4.0 International license.

\section{Tables}

Table 1: Highly downregulated genes in SCVs relative to NHMuc

\begin{tabular}{|c|c|c|c|c|}
\hline Gene ID & $\begin{array}{c}\text { Fold } \\
\text { Change }^{\mathrm{a}}\end{array}$ & p-Value ${ }^{b}$ & Protein description & $\begin{array}{c}\text { PAO1 } \\
\text { gene ID }\end{array}$ \\
\hline NHmuc_01025 & -75 & $2.6 \times 10^{-11}$ & Putative acetyltransferase & PA4364 \\
\hline $\operatorname{mexC}$ & -53 & $1.1 \times 10^{-11}$ & RND multidrug efflux fusion protein MexC & PA4599 \\
\hline ibpA & -31 & $1.1 \times 10^{-7}$ & Heat-shock protein IbpA & PA3126 \\
\hline $\operatorname{algD}$ & -30 & $1.0 \times 10^{-9}$ & GDP-mannose 6-dehydrogenase AlgD & PA3540 \\
\hline NHmuc_05385 & -29 & $8.5 \times 10^{-8}$ & $17 \mathrm{kDa}$ surface antigen & PA5182 \\
\hline NHmuc_04138 & -29 & $1.3 \times 10^{-10}$ & Periplasmic metal-binding protein & PA3520 \\
\hline $\operatorname{grpE}$ & -25 & $1.2 \times 10^{-7}$ & Heat shock protein GrpE & PA4762 \\
\hline htpG & -23 & $3.6 \times 10^{-7}$ & Heat shock protein 90 & PA1596 \\
\hline NHmuc_0100 & -23 & $1.8 \times 10^{-5}$ & Hypothetical, unclassified, unknown & \\
\hline $\mathrm{clpB}$ & -21 & $6.5 \times 10^{-6}$ & ClpB protein & PA4542 \\
\hline nfxB & -21 & $3.7 \times 10^{-7}$ & Transcriptional regulator $\mathrm{Nf} \times \mathrm{B}$ & PA4600 \\
\hline fsxA & -18 & $7.9 \times 10^{-8}$ & FxsA protein & PA4387 \\
\hline NHmuc_04950 & -18 & $3.3 \times 10^{-6}$ & Molecular chaperone DnaK & PA4761 \\
\hline NHmuc_05744 & -17 & $5.8 \times 10^{-5}$ & Putative lipoprotein & PA5526 \\
\hline dapB & -17 & $2.8 \times 10^{-6}$ & Dihydrodipicolinate reductase & PA4759 \\
\hline hsIV & -17 & $4.1 \times 10^{-7}$ & ATP-dependent protease subunit & PA5053 \\
\hline NHmuc_01173 & -16 & $4.7 \times 10^{-6}$ & Hypothetical protein & PA2756 \\
\hline NHmuc_01024 & -16 & $6.1 \times 10^{-7}$ & Putative transporter & PA4365 \\
\hline NHmuc_04180 & -16 & $3.3 \times 10^{-6}$ & Recombinase A & PA3617 \\
\hline $\operatorname{mexD}$ & -15 & $9.5 \times 10^{-7}$ & RND multidrug efflux transporter MexD & PA4598 \\
\hline NHmuc_04776 & -15 & $6.1 \times 10^{-6}$ & PAS/PAC sensor signal transduction histidine kinase & PA4197 \\
\hline rsmA & -15 & $7.5 \times 10^{-4}$ & Carbon storage regulator & PA0905 \\
\hline NHmuc_01174 & -14 & $3.3 \times 10^{-7}$ & Hypothetical protein & PA0737 \\
\hline NHmuc_01594 & -13 & $2.1 \times 10^{-5}$ & Putative oxidoreductase & PA1137 \\
\hline mucA & -13 & $7.4 \times 10^{-5}$ & Anti-sigma factor MucA & PA0763 \\
\hline NHmuc_03262 & -12 & $2.5 \times 10^{-9}$ & Hypothetical protein & PA3505 \\
\hline NHmuc_04386 & -12 & $6.8 \times 10^{-5}$ & Surface antigen & PA3819 \\
\hline amrZ & -12 & $3.0 \times 10^{-5}$ & Alginate and motility regulator $\mathrm{Z}$ & PA3385 \\
\hline$g \ln A$ & -12 & $5.8 \times 10^{-6}$ & Gutamine synthetase & PA5119 \\
\hline groES & -12 & $3.9 \times 10^{-5}$ & Co-chaperonin GroES & PA4386 \\
\hline dnaJ & -12 & $7.5 \times 10^{-5}$ & Chaperone protein DnaJ & PA4760 \\
\hline algu & -11 & $1.8 \times 10^{-4}$ & RNA polymerase sigma factor AlgU & PA0762 \\
\hline $\operatorname{clpX}$ & -11 & $4.5 \times 10^{-4}$ & ATP-dependent protease subunit CIpX & PA1802 \\
\hline NHmuc_04521 & -11 & $1.9 \times 10^{-6}$ & Periplasmic ligand-binding sensor & PA3952 \\
\hline
\end{tabular}

a The magnitude of gene expression (fold change) was determined by comparing transcription in three replicates of $\mathrm{NH}$ with that in three replicates each of the two SCV strains.

${ }^{b} p$-values were assessed by performing an EDGE test using CLC software. 
bioRxiv preprint doi: https://doi.org/10.1101/356386; this version posted July 9,2018 . The copyright holder for this preprint (which was not certified by peer review) is the author/funder, who has granted bioRxiv a license to display the preprint in perpetuity. It is made available under aCC-BY 4.0 International license.

Table 2: Highly upregulated genes in SCVs relative to NHMuc

\begin{tabular}{|c|c|c|c|}
\hline Gene ID & $\begin{array}{c}\text { Fold } \\
\text { Change }^{\mathrm{a}}\end{array}$ & p-Value & Protein description \\
\hline NHmuc_04720 & 62 & $3.2 \times 10^{-16}$ & Hypothetical protein \\
\hline $\operatorname{trxB2}$ & 55 & $4.0 \times 10^{-16}$ & Thioredoxin reductase 2 \\
\hline NHmuc_01300 & 35 & $3.7 \times 10^{-15}$ & Putative alkyl hydroperoxide reductase \\
\hline NHmuc_02350 & 28 & $1.4 \times 10^{-26}$ & Putative acyl carrier protein \\
\hline ahpF & 28 & $8.4 \times 10^{-13}$ & Alkyl hydroperoxide reductase subunit F \\
\hline kata & 23 & $7.1 \times 10^{-10}$ & Catalase \\
\hline NHmuc_03862 & 20 & $6.9 \times 10^{-21}$ & Putative ankyrin domain-containing protein \\
\hline NHmuc_03863 & 19 & $1.8 \times 10^{-18}$ & Putative hydrolase \\
\hline NHmuc_00257 & 19 & $1.4 \times 10^{-12}$ & Putative CBS domain protein \\
\hline ahpC & 18 & $9.0 \times 10^{-8}$ & Alkyl hydroperoxide reductase subunit C \\
\hline chiC & 17 & $1.5 \times 10^{-9}$ & Chitinase \\
\hline NHmuc_04185 & 17 & $6.7 \times 10^{-6}$ & RNA polymerase sigma factor RpoS \\
\hline aprA & 12 & $5.1 \times 10^{-8}$ & Alkaline metalloproteinase \\
\hline NHmuc_04924 & 11 & $3.2 \times 10^{-16}$ & CsbD family protein \\
\hline NHmuc_04718 & 11 & $1.5 \times 10^{-11}$ & Hypothetical protein \\
\hline NHmuc_04925 & 11 & $2.2 \times 10^{-8}$ & Transport-associated \\
\hline NHmuc_00127 & 10 & $5.9 \times 10^{-7}$ & Putative hemolysin \\
\hline Snr1 & 10 & $4.9 \times 10^{-7}$ & Cytochrome c Snr1 \\
\hline lecB & 9 & $5.9 \times 10^{-8}$ & Fucose-binding lectin PA-IIL \\
\hline NHmuc_03413 & 8 & $9.1 \times 10^{-15}$ & Phage terminase, small subunit \\
\hline katB & 8 & $9.1 \times 10^{-6}$ & Catalase \\
\hline NHmuc_04074 & 8 & $2.2 \times 10^{-12}$ & Leucyl-tRNA synthetase \\
\hline phzG2_2 & 7 & $3.3 \times 10^{-6}$ & Pyridoxamine 5'-phosphate oxidase \\
\hline NHmuc_03358 & 7 & $2.0 \times 10^{-8}$ & Putative protein associated inclusion bodies \\
\hline phzE1_1 & 7 & $3.2 \times 10^{-6}$ & Phenazine biosynthesis protein PhzE \\
\hline NHmuc_00055 & 7 & $6.1 \times 10^{-7}$ & Hypothetical protein \\
\hline NHmuc_01628 & 7 & $1.3 \times 10^{-8}$ & Hypothetical protein \\
\hline cbpD & 7 & $4.1 \times 10^{-6}$ & Chitin-binding protein CbpD \\
\hline NHmuc_00546 & 6 & $2.0 \times 10^{-3}$ & LysR transcriptional regulator \\
\hline rhlR & 6 & $1.2 \times 10^{-4}$ & Transcriptional regulator RhIR \\
\hline gcdH & 6 & $4.3 \times 10^{-5}$ & Glutaryl-CoA dehydrogenase \\
\hline NHmuc_01422 & 6 & $4.1 \times 10^{-4}$ & Putative dna-binding stress protein \\
\hline NHmuc_04078 & 6 & $1.5 \times 10^{-10}$ & Oxidoreductase probably involved in sulfite reduction \\
\hline rsaL & 6 & $1.2 \times 10^{-2}$ & Regulatory protein RsaL \\
\hline
\end{tabular}

a The magnitude of gene expression (fold change) was determined by comparing transcription in three replicates of $\mathrm{NH}$ with that in three replicates each of the two SCV strains.

${ }^{b} \mathrm{p}$-values were assessed by performing an EDGE test using CLC software. 


\section{Figures}

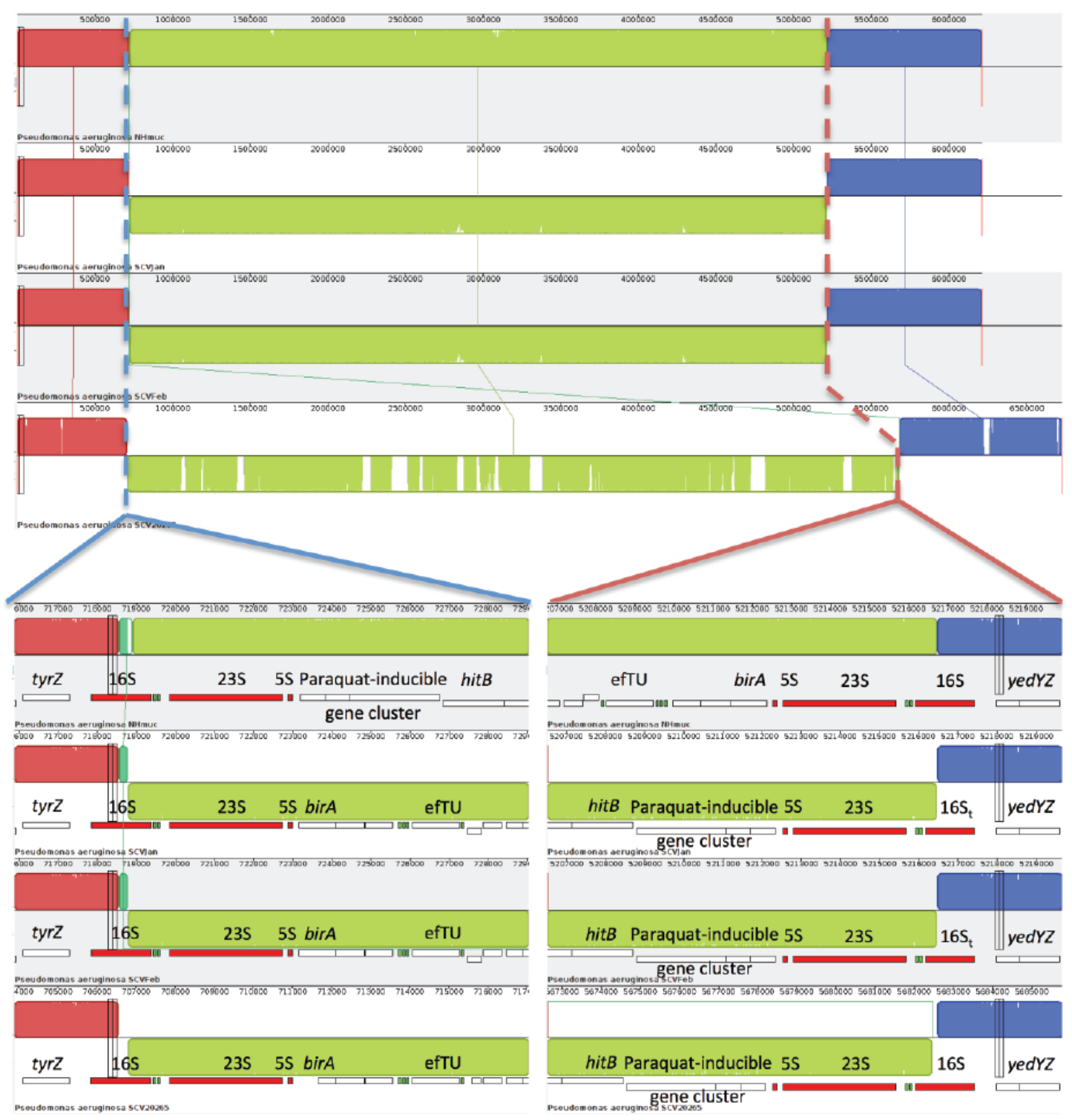

Figure 1. A common large scale chromosomal inversion in three $P$. aeruginosa strains is the genetic basis of conversion to the SCV phenotype. From top to bottom strains NHMuC, SCVJan, SCVFeb and SCV20265 are displayed. Dashed lines indicate the inversion breakpoints present in the 16S rRNA genes. An inversion with highly similar breakpoints is present in the genome of strain SCV20265 a SCV isolated from a patient with CF. Within strains SCVJan and SCVFeb a unique truncated version of the $16 \mathrm{~S}$ rRNA gene $\left(16 \mathrm{~S}_{\mathrm{t}}\right)$ could be resolved, which could not be detected in strain SCV20265. 
a)

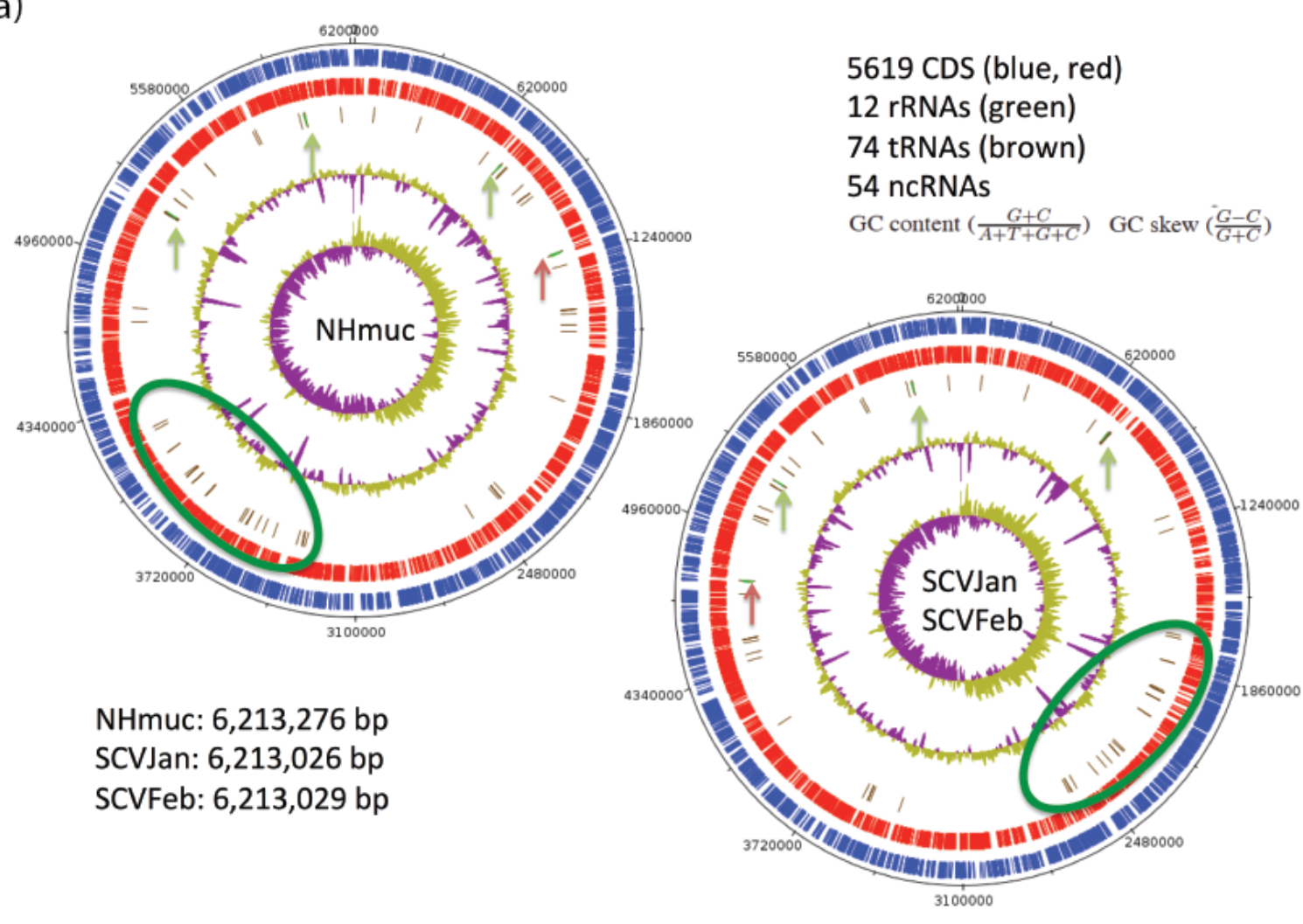

b)

Figure 2. Chromosomal maps of $P$. aeruginosa NHmuc (a) and SCVJan/SCVFeb (b). The circular genomes of both strains are shown. Genomes of both SCV strains are 250 bp smaller compared to the parental strain NHmuc. Exact genome sizes are given in lower left corner. In blue (circle1) genes lying on the forward strand are shown and in red (circle 2) those on the reverse strand. In circle 3 tRNA genes are shown in brown, often clustered together with green rRNA genes, which have been additionally marked by vertical arrows. The red arrow shows the transposition of rRNA operon 3 in addition to that of a large tRNA region (green ellipse) due to the described chromosomal inversion. Circle 4 shows the GC content, whereas in circle 5 a GC skew is shown. Number of CDS, rRNAs, tRNAs and ncRNAs are identical in all strains (upper right corner according to GenBank submission). This map has been created using DNAplotter ${ }^{72}$ (Carver et al., 2009). 


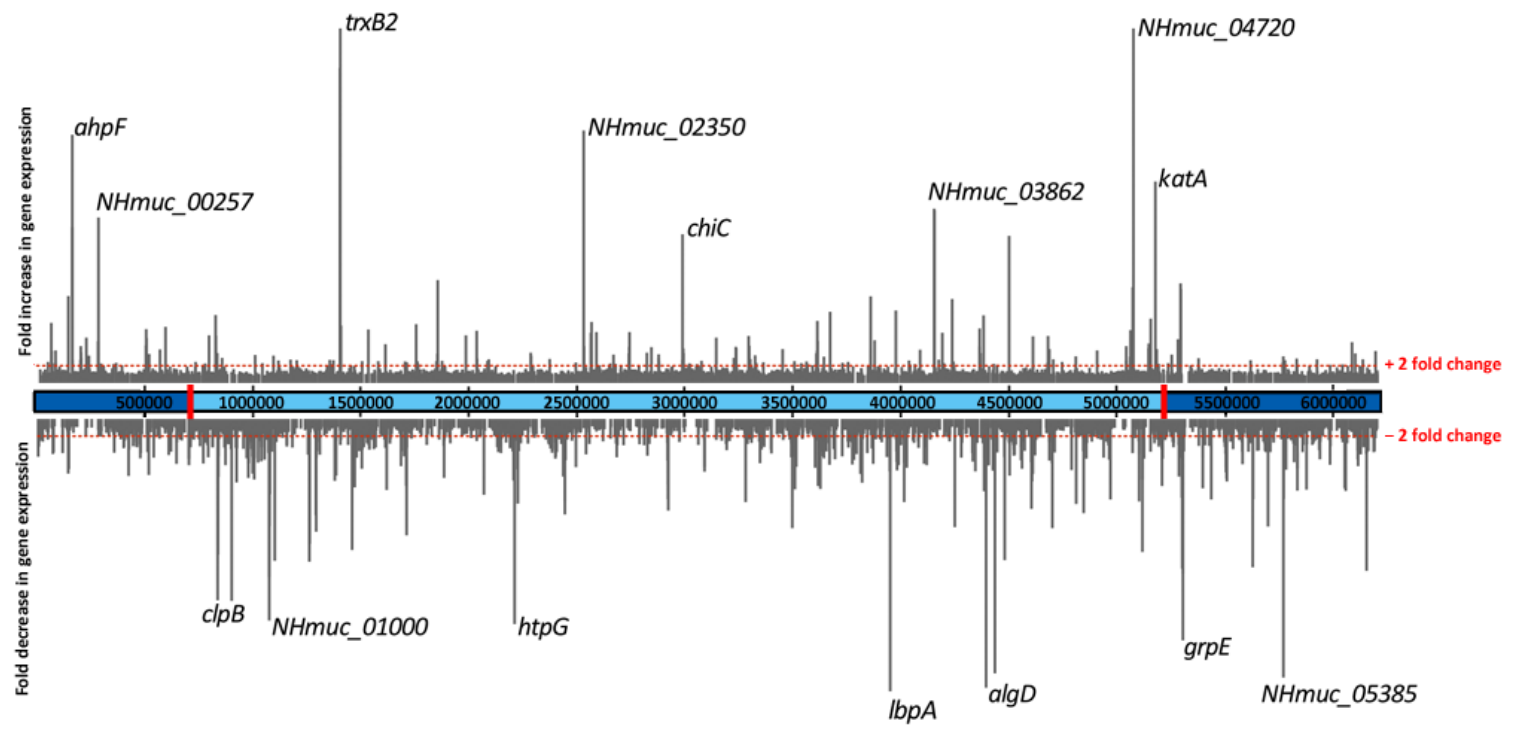

Figure 3. Global changes in gene transcription on conversion to the SCV phenotype. Foldchanges in gene expression for SCVJan/Feb relative to NHMuc are shown in the context of the NHMuc genome. Breakpoints that define the genomic inversion present in SCVJan and SCVFeb are indicated by red rectangles. 
a

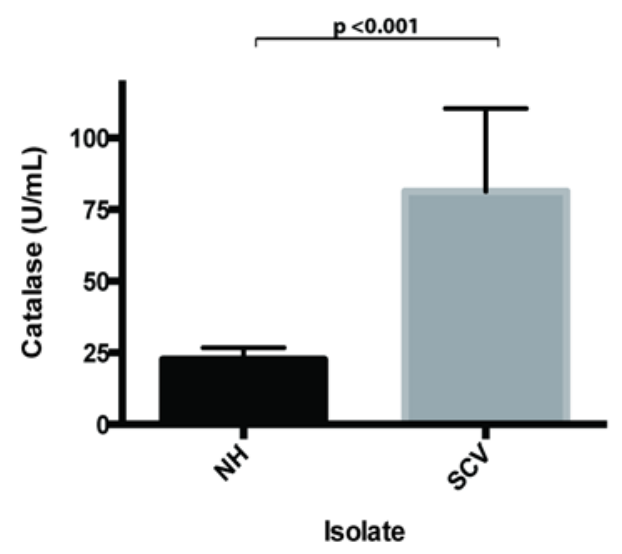

b

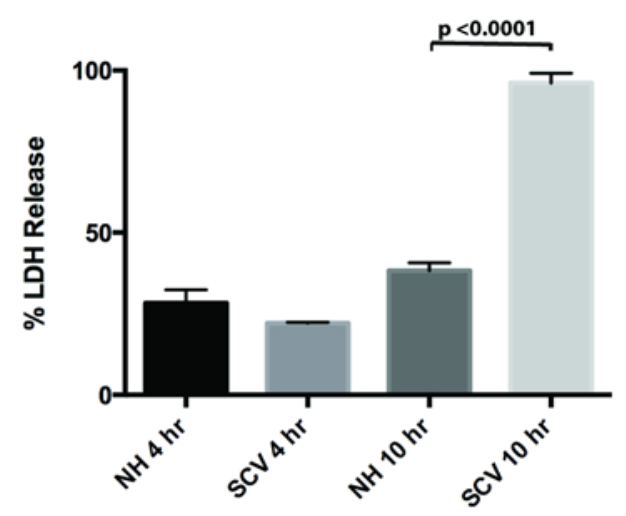

C

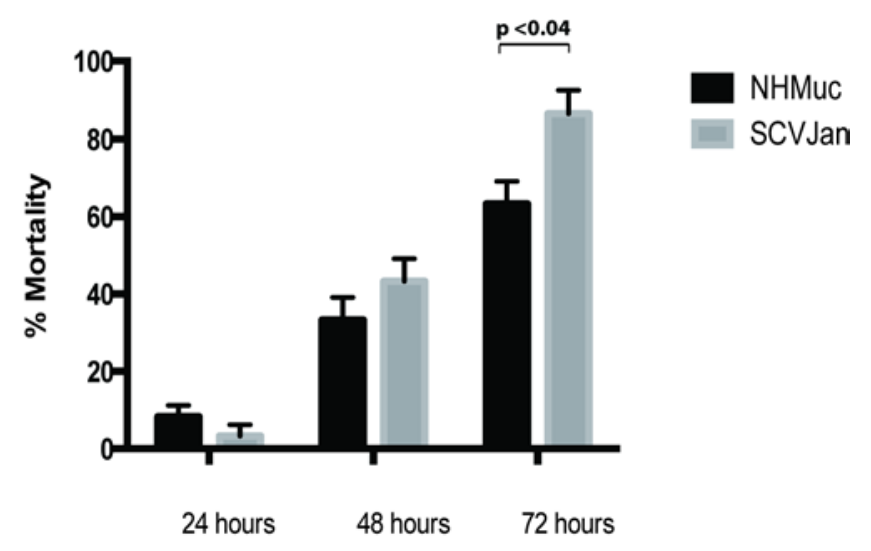

Figure 4. Phenotypic characterisation of SCVJan. a) Catalase activity assay demonstrating marked increase in catalase activity in the SCV as compared to the $\mathrm{NH}$ parent strain. b) \% LDH released from macrophage cell line with comparison between NHMuC and SCVJan over a 4 and 10 hour time period. c) Galleria mellonella larvae survival over time when infected with the NHMuc and SCVJan strains monitored over 72 hours. 


\section{Supplementary Figure}

a

\author{
Mucoid strain
}

PCR1

SCV strain

PCR2

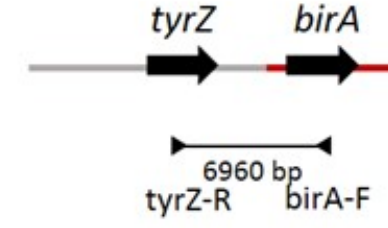

b

MW
MW

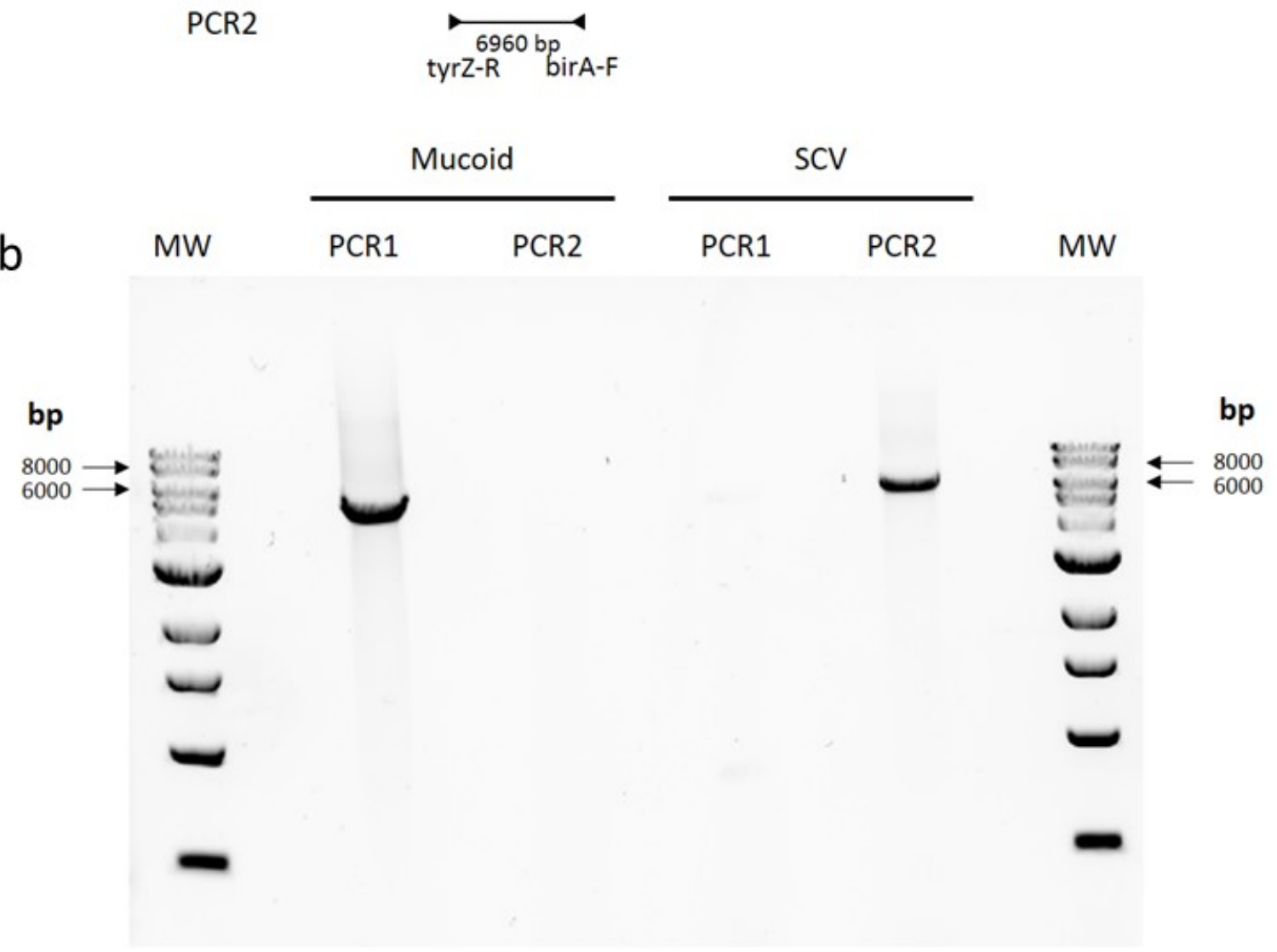

birA yedz

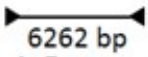

birA-F yedZ-R

yedz

Figure S1. The mucoid strain, NHMucJan, isolated from the same chronic infection model as SCVJan does not contain a similar genome inversion. a) To determine if NHMucJan contains a similar genome inversion as SCVJan and SCVFeb we developed a PCR strategy using primers specific to $t y r Z$ or $y e d Z$ which lie outside and adjacent to the inverted region in combination with primer specific to birA, which is adjacent to the rRNA genes within the inverted region. Only the primer pair expected to give a PCR product for each strain is shown. The inverted sequence in the SCV strain is highlighted in red. b) Consistent with the hypothesis that genome inversion drives conversion we observed a PCR product only with birA-F/yedZ-R primer pair for NHMucJan indicating that the genome structure in this region is identical to the parent strain NHMuc. 